\title{
Lattice Distortion Effects on the Magnetostructural Phase Transition of MnAs
}

\author{
F. Iikawa, ${ }^{1, *}$ M. J. S. P. Brasil, ${ }^{1}$ C. Adriano, ${ }^{1}$ O. D. D. Couto, ${ }^{1}$ C. Giles, ${ }^{1,2}$ P. V. Santos, ${ }^{3}$ \\ L. Däweritz, ${ }^{3}$ I. Rungger, ${ }^{4}$ and S. Sanvito ${ }^{4}$ \\ ${ }^{1}$ Instituto de Física "Gleb Wataghin," UNICAMP, Campinas-SP, C.P. 6165, 13083-970, Brazil \\ ${ }^{2}$ Laboratório Nacional de Luz. Síncrotron, C.P. 6192, 13084-971 Campinas-SP, Brazil \\ ${ }^{3}$ Paul-Drude-Institut für Festkörperelektronik, Hausvogteiplatz, 5-7, 10117 Berlin, Germany \\ ${ }^{4}$ Department of Physics, Trinity College, Dublin 2, Ireland
}

(Received 22 March 2005; published 11 August 2005)

\begin{abstract}
We present a systematic experimental and theoretical study of the first-order phase transition of epitaxially grown MnAs thin films under biaxial tensile stress. Our results give direct information on the dependence of the phase-transition temperature of MnAs films on the lattice parameters. We demonstrate that an increase of the lattice constant in the hexagonal plane raises the phase-transition temperature $\left(T_{\mathrm{p}}\right)$, while an increase of the perpendicular lattice constant lowers $T_{\mathrm{p}}$. The results of calculations based on density functional theory are in good agreement with the experimental ones. Our findings open exciting prospects for magneto-mechanical devices, where the critical temperature for ferromagnetism can be engineered by external stress.
\end{abstract}

PACS numbers: 75.70.Ak, 61.50.Ks, 68.60.-p

MnAs presents a first-order phase transition at $\sim 40^{\circ} \mathrm{C}$, changing from ferromagnetic/hexagonal $(\alpha$-phase NiAs structure) to paramagnetic/orthorhombic ( $\beta$-phase $\mathrm{MnP}$ structure) [1]. This magneto-structural phase transition has important implications for technological applications. The magneto-elastic effects are useful for transducers [2], while their magneto-caloric properties are interesting for developing refrigeration devices [3]. In recent years, the attention given to MnAs has been strongly amplified by the possibility of epitaxial growth on GaAs substrates [4]. The integration of ferromagnetic materials with semiconductors is a subject of great interest for spintronics, and MnAs grown on GaAs is a strong candidate for spin injection devices [5].

From the theoretical point of view, the treatment of the first-order phase transition of materials with magnetoelastic properties is a rather complex issue. Early simple phenomenological thermodynamic treatments based on the localized Heisenberg model [1] have been used to explain the properties of MnAs under an external hydrostatic pressure and magnetic field. Sophisticated band structure calculations are required for a precise quantitative analysis, although in this case it is difficult to introduce a statistical treatment to describe a first-order phase transition [6].

We present an experimental and theoretical investigation of the magneto-structural phase transition of MnAs films grown on GaAs. Those films present a nonabrupt phase transition with the coexistence [7] of the two phases in form of periodically alternating stripes [8,9] for a large temperature range $\left(\sim 20^{\circ} \mathrm{C}\right)[7-10]$. As a result of this phase coexistence a considerable fraction of the volume of the MnAs epitaxial films is usually in the paramagnetic phase at $\sim 30^{\circ} \mathrm{C}$, which is a strong limitation for room temperature spintronic devices. The growth of MnAs films on different crystal orientations has been suggested as an alternative that can provide higher phase-transition tem- peratures [10]. The detailed mechanism that associates the crystal distortion (lattice parameter variation) with the phase-transition temperature is, however, still unclear, This issue was addressed in the early days by Menyuk et al. [11]. Recently, sophisticated ab initio methods pointed out the important role played by the $p$ - $d$ hybridization as the driving force of the MnAs first-order phase transition [12]. However, detailed calculations correlating the magneto-elastic first-order phase transition with the lattice parameter variation have not yet been reported.

In order to get more insight into the MnAs phase transition, we have designed an $\mathrm{x}$-ray diffraction experiment and performed theoretical calculations to investigate in detail the dependence of the phase-transition temperature on crystal distortions. Experimental results show a clear variation of the phase-transition temperature of $\mathrm{MnAs}$ films due to the lattice distortions imposed by the substrate and by the application of an external biaxial stress. We observed that an increase of the unit cell volume does not necessarily result in an increase of the transition temperature, in contrast to the phenomenological model of Bean and Rodbell [1]. From accurate electronic structure calculations, we obtained a general and quantitative relationship between the exchange energy and the independent variation of each MnAs lattice parameter. We propose that the variation of the exchange energy can be associated with the MnAs phase-transition temperature. Based on this assumption, we were able to obtain a direct relationship between the phase-transition temperature and any MnAs lattice distortion, even though our calculations do not include a thermodynamic treatment of the phase transition. The validity of our approximation is corroborated by a good agreement between the experimental results and the theoretical predictions.

Our MnAs films are epitaxially grown on $\mathrm{GaAs}(001)$ by molecular beam epitaxy under growth conditions that yield 


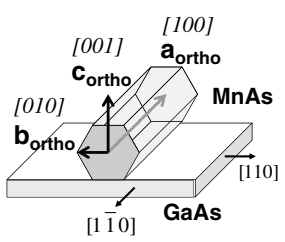

(a)

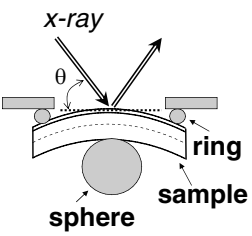

(b)
FIG. 1. (a) Schematic diagram of a unit cell of the $A$-orientation MnAs/GaAs(001). The lattice parameters $a_{\text {ortho }}$, $b_{\text {ortho }}$, and $c_{\text {ortho }}$ are defined in the orthorhombic structure notation. (b) Diagram of a biaxial stress ring cell. The x-ray beam was focused at the center of the ring.

$A$-oriented films, [13] as shown in the Fig. 1(a). We use here the orthorhombic notation [see Fig. 1(a)], which is more appropriate for MnAs film on GaAs, since the films are deformed by the substrate and lose the hexagonal symmetry to an orthorhombic one even in the $\alpha$ phase. The results presented here were obtained for a $130 \mathrm{~nm}$ thick MnAs film. External biaxial strain was applied using a stress ring cell [14] assembled in a temperature controlled continuous $\mathrm{N}_{2}$ gas flow cryostat. The sample is mounted between a ring and a sphere [Fig. 1(b)] and biaxial strain is achieved by moving the sphere towards the ring [14]. The film (much thinner than the substrate), which is located on the upper side of the sample, undergoes a symmetric biaxial tensile strain at the center of the ring. The strain is approximately uniform within $\sim 1 \mathrm{~mm}$ of diameter from the center, which is of the order of the x-ray beam spot.

Figure 2(a) shows the x-ray diffraction patterns for the $130 \mathrm{~nm}$ MnAs film under different external stress conditions at $T=0{ }^{\circ} \mathrm{C}$. The diffraction peak corresponds to the $\operatorname{MnAs}(004)$ reflection. When an external biaxial tensile strain is applied to the MnAs films, the x-ray diffraction peak shifts to higher angles, indicating a decrease of the lattice parameter along the growth direction, due to the stretching of the in-plane parameters. We also note a decrease of the x-ray intensity for increasing strain. This is attributed to a decrease of the probed volume, which

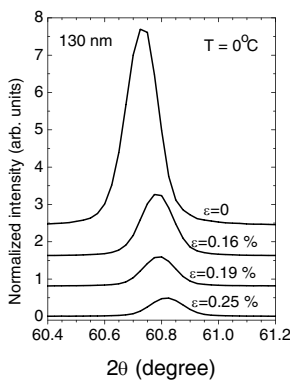

(a)

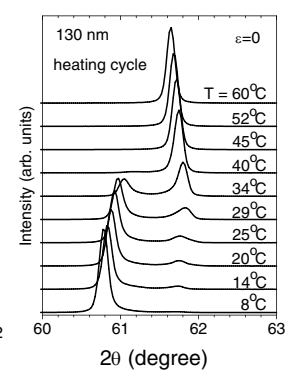

(b)
FIG. 2. (a) Biaxial tensile strain dependence of the x-ray diffraction patterns for the MnAs/GaAs film. (b) Temperature dependent $\mathrm{x}$-ray diffraction patterns for the same sample without an external strain. maintains the geometrical condition for a maximum of diffraction as the sample curvature changes. We assumed that the variation of the scattering structure factor with strain is negligible since the strain range used in this work is relatively small (less than $0.3 \%$ ).

Figure 2(b) shows the $\mathrm{x}$-ray diffraction spectra obtained for temperatures ranging from zero to $60^{\circ} \mathrm{C}$ during a heating cycle without an external strain. The diffraction patterns show two peaks: the lower one at $2 \theta \sim 61^{\circ}$ corresponds to the $\alpha$-MnAs phase and the peak at $2 \theta \sim 62^{\circ}$ corresponds to the $\beta$-MnAs phase. At intermediate temperatures, x-ray spectra exhibit both peaks, indicating the coexistence of the two phases. Measurements for cooling cycles give similar results with a small shift of the phase transition to lower temperatures, resulting in a thermal hysteresis, as observed in previous works [10].

Figure 3 shows the volume fraction $F_{\alpha}$ vs temperature of the $\alpha$-MnAs phase, with and without an external strain. The fraction $F_{\alpha}$ was obtained from the ratio of the integrated intensity of the $\alpha$ phase x-ray diffraction peak relative to the sum of the total intensity of the peaks for both $\alpha$ and $\beta$ phases. $F_{\alpha}$ presents a nonabrupt profile with a thermal hysteresis, as discussed before. Note that the phase transition of the films starts at temperatures smaller than that of the transition temperature of the bulk $\left(40^{\circ} \mathrm{C}\right)$. When an external tensile strain is applied, the curves show a clear shift to higher temperatures. We also observed very similar results for a $160 \mathrm{~nm} \mathrm{MnAs} / \mathrm{GaAs}$ film. Because of the phase coexistence across a large range of temperatures, the phase-transition temperature is not well defined for the film. Therefore, we choose to define a characteristic transition temperature, $T_{\mathrm{p}}$, as the temperature where $F_{\alpha}$ is 0.5 .

We present in Table I the lattice parameters of the MnAs film, as grown and under external strain, and those of bulk MnAs from the literature [15]. The lattice parameters of the as-grown film were obtained from specular and grazing incident diffraction (GID) measurements. In the case of the film under an external strain, we have only measured the parameter $c_{\text {ortho }}$, since the biaxial stress cell does not allow GID measurements. In this case, we have derived the other parameters using the relation between the in-

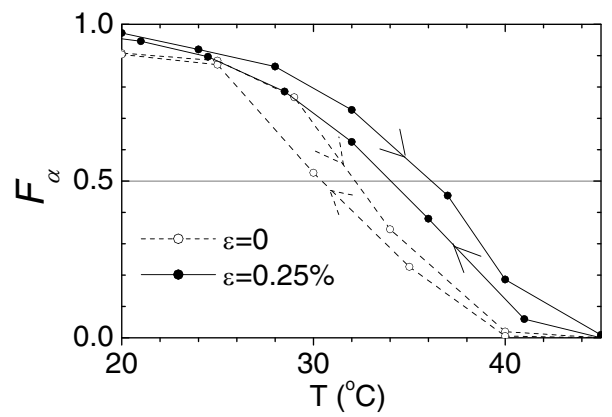

FIG. 3. Temperature dependence of the volume fraction of $\alpha$-phase $F_{\alpha}$ of the $130 \mathrm{~nm} \mathrm{MnAs} \mathrm{film.}$ 
TABLE I. Lattice parameters, unit cell volume $(V)$, and relative temperature variation $\frac{\delta T_{P}}{T_{P}}$ of the $130 \mathrm{~nm}$ MnAs film and of MnAs bulk [15] in the $\alpha$-MnAs phase for $T=20^{\circ} \mathrm{C}$. In parenthesis the lattice parameter variation relative to bulk is shown. $\frac{\delta J_{0}}{J_{0}}$ are theoretical data.

\begin{tabular}{|c|c|c|c|}
\hline & MnAs Bulk & $\begin{array}{c}\text { film } \\
\epsilon_{\|}=0\end{array}$ & $\begin{array}{c}\text { film } \\
\epsilon_{\|}=0.25 \%\end{array}$ \\
\hline$a_{\text {ortho }}(\AA)$ & 5.706 & $5.764(+1.0 \%)$ & $5.778(+1.27 \%)$ \\
\hline$b_{\text {ortho }}(\AA)$ & 3.724 & $3.710(-0.37 \%)$ & $3.719(-0.13 \%)$ \\
\hline$c_{\text {ortho }}(\AA)$ & 6.450 & $6.440(-0.15 \%)$ & $6.434(-0.25 \%)$ \\
\hline$V\left(\AA^{3}\right)$ & 137.06 & $137.72(+0.48 \%)$ & $138.26(+0.88 \%)$ \\
\hline$\frac{\delta T_{P}}{T_{0}}(\%)$ & 0.0 & -3.2 & -1.6 \\
\hline$\frac{\delta f_{0}}{J_{0}}(\%)$ & 0.0 & -3.0 & -2.4 \\
\hline
\end{tabular}

plane $\left(\epsilon_{\|}=\frac{\Delta a_{\text {ortho }}}{a_{\text {ortho }}}=\frac{\Delta b_{\text {ortho }}}{b_{\text {ortho }}}\right)$ and out-of-plane $\left(\epsilon_{z}=\frac{\Delta c_{\text {ortho }}}{c_{\text {ortho }}}\right)$ lattice parameter variations given by $\epsilon_{\|}=-\left[\frac{C_{12}+C_{23}}{C_{22}}\right] \epsilon_{z}$, where $C_{i j}$ are the elements of the elastic constant tensor of MnAs [16]. For the condition of maximum strain applied to the sample we obtained from the x-ray measurements $\frac{\Delta c_{\text {ortho }}}{c_{\text {ortho }}}=0.11 \%$, which corresponds to $\frac{\Delta a_{\text {ortho }}}{a_{\text {ortho }}}=\frac{\Delta b_{\text {ortho }}}{b_{\text {ortho }}}=0.25 \%$. Comparing the lattice parameters between bulk and as-grown film, we observe that the MnAs film is stretched along the $a_{\text {ortho }}$ direction and is shrunk in the hexagonal basal plane. Similar results were observed in a previous work [10]. The resulting unit cell volume in the film is $0.48 \%$ larger than that in the bulk and presents a smaller $T_{\mathrm{p}}$. When the film is under external tensile strain, the unit cell volume shows a further increase (becoming $0.88 \%$ larger than the bulk). In this case, however, the increase of the cell volume is accompanied by an increase of $T_{\mathrm{p}}$. Bean and Rodbell [1] have predicted an enhancement of $T_{\mathrm{p}}$ upon increasing the MnAs unit cell volume. Our results show, however, that $T_{\mathrm{p}}$ is not a simple function of the cell volume and it does depend on the lattice parameters in a more complex way.

In order to analyze our results, we have performed calculations based on the density functional theory (DFT) using the numerical implementation contained in the pseudopotential code SIESTA [17], and considering the generalized gradient approximation of the exchange correlation potential [18]. We used an $s-p$ - $d$ basis set for both Mn and As with double zeta polarized $s$ and $p$ orbitals and simple double zeta for the $d$ orbitals. The cutoff radii of the first zetas are as follows: Mn $4 s$ and $4 p 7.5$ bohr, Mn 3d 5.5 bohr, As $4 s, 4 p$, and $4 d 6$ bohr. The second zeta cutoffs are determined with the split norm scheme [17]. Finally the real space mesh cutoff is $300 \mathrm{Ry}$ and we considered $616 k$ points in the full B31 Brillouin zone. We analyzed several possible magnetic configurations for each lattice distortion, evaluating a Heisenberg-type expression for the total energy: $E_{\mathrm{H}}=E_{0}-\frac{1}{2} \Sigma_{i, j} J_{i j} \mathbf{s}_{i} \cdot \mathbf{s}_{j}$, where $J_{i j}$ are the Heisenberg exchange parameters $\left(J_{i i}=0\right), E_{0}$ is the total energy of the paramagnetic state and $\mathbf{s}_{i}$ is the spin unit vector at site $i$. The coefficient $J_{i j}$ depends solely on the separation vector $\mathbf{R}_{i, j}$ between spins $i$ and $j$. The parameters $J_{(i, j)}$ were obtained by minimizing the difference between the total energy obtained from the DFT calculations and the Heisenberg energy, where couplings up to third nearest neighbors were considered. A detailed description of the calculations and of the fitting procedure will be presented elsewhere [19]. The parameter $J_{0}=\Sigma_{j} J_{0 j}$, which in the mean field approximation is proportional to the Curie temperature, is a measure of the stability of the ferromagnetic phase $[1,19,20]$. The larger the gain in magnetic energy, the higher should be $T_{\mathrm{p}}$. As a first approximation, we assume that the change of $T_{\mathrm{p}}$ is proportional to the equivalent change of $J_{0}: \frac{\delta T_{\mathrm{p}}}{T_{\mathrm{p}}}=\frac{\delta J_{0}}{J_{0}}$. Table I gives the calculated values of $\frac{\delta J_{0}}{J_{0}}$ for the film, with and without an external stain, and its relative change with respect to the bulk. We observe that the calculated values of $\frac{\delta J_{0}}{J_{0}}$ are in very good agreement with the corresponding experimental values of $\frac{\delta T_{\mathrm{p}}}{T_{\mathrm{p}}}$ for the as-grown sample. The agreement is not equally good for the film under external biaxial strain. In this case the in-plane parameters were calculated from the out-of-plane one using the elastic tensor, which carries a significant experimental uncertainty. We remark, in particular, that the theory correctly predicts the direction of the transition temperature changes due to the film distortions: a decrease of $T_{\mathrm{p}}$ from bulk to the as-grown film and a partial recovery of $T_{\mathrm{p}}$ when a biaxial tensile strain is applied to the film.

In order to accomplish a more general analysis, we may consider that for small distortions $J_{0}$ is linearly dependent on the orthorhombic lattice parameters [same notation of the Fig. 1(a)]:

$$
\frac{\delta J_{0}}{J_{0}}=K_{a} \frac{\delta a}{a}+K_{b} \frac{\delta b}{b}+K_{c} \frac{\delta c}{c},
$$

where $K_{a}, K_{b}$, and $K_{c}$ are constants that can be determined by calculating $J_{0}$ for various distorted cells and fitting the results using the linear relationship expressed above. Following this procedure we observed a well-behaved linear dependence from which we obtained:

$$
K_{a}=-1.0, \quad K_{b}=5.2, \quad K_{c}=4.2
$$

The results clearly show that stretching the unit cell along the basal plane raises $T_{\mathrm{p}}$ (since $K_{b}$ and $K_{c}>0$ ), while stretching along the $a$ axis lowers $T_{\mathrm{p}}\left(K_{a}<0\right)$. We point out that the distortion of a single lattice parameter alters the value of various distances between atoms in a complex way, so that the constants $K_{i}$ reflect the combination of all those changes. We observed that an increase of the separation between $\mathrm{Mn}$ atoms (including the distance between nearest $\mathrm{Mn}$ neighbors and the $\mathrm{Mn}-\mathrm{Mn}$ separation in the 
basal plane) results in an increase of $T_{\mathrm{p}}$, while an increase of the separation between $\mathrm{Mn}$ and As atoms results in a decrease of $T_{\mathrm{p}}$ [19]. When the cell is stretched along the $a_{\text {ortho }}$ axis, the increase of the Mn-As separations slightly dominates, resulting in a small negative value for $K_{a}$. For distortions along the $b_{\text {ortho }}$ and $c_{\text {ortho }}$ axes, the increase of the Mn-Mn separation strongly dominates so that $K_{b}$ and $K_{c}$ are positive.

We may now interpret our experimental results. The strain effects of growing a MnAs thin film with the $A$ orientation on GaAs results in the stretching of the $a_{\text {ortho }}$ axis and the contraction of both the $b_{\text {ortho }}$ and $c_{\text {ortho }}$ axes. All these distortions contribute to reduce $T_{\mathrm{p}}$. When an external biaxial stress is applied along the film plane, the lattice parameters $a_{\text {ortho }}$ and $b_{\text {ortho }}$ increase while the $c_{\text {ortho }}$ parameter decreases. The net result is a small enhancement of $T_{\mathrm{p}}$, reflecting the balance of the $K$ parameters and the relative large value of $K_{b}$. Our calculations also explain why a hydrostatic pressure lowers the phase-transition temperature of bulk MnAs, as observed in previous works [1]. Under hydrostatic pressure, the $b_{\text {ortho }}$ and $c_{\text {ortho }}$ parameters suffer a stronger contraction than $a_{\text {ortho }}$ due to the anisotropy of the MnAs elastic constant tensor [16]. This effect, combined with the fact that $\left|K_{b}\right|$ and $\left|K_{c}\right|$ are bigger than $\left|K_{a}\right|$, results in a decrease of $T_{\mathrm{p}}$.

In summary, based on our results, we propose that higher $T_{\mathrm{p}}$ can be achieved by growing MnAs in such a way to maximize the lattice parameters of the basal plane and minimize the lattice parameter in the perpendicular direction. Finally, the possibility of moving across the phasetransition temperature by applying an external biaxial pressure opens a new avenue to mechanically driven magneto-devices. One can envision composite materials comprising MnAs and piezoelectric compounds, where the magnetism can be mechanically switched on and off.

We thank C. Hermann and M. Kästner for the sample growth, F. Cedeira for helpful discussions on the manuscript, and FAPESP, CAPES, CNPq, and SFI (Grant No. SFI02/IN1/I175) for financial support.

*Electronic address: iikawa@ifi.unicamp.br

[1] C. P. Bean and D. S. Rodbell, Phys. Rev. 126, 104 (1962).

[2] V. A. Chernenko et al., J. Appl. Phys. 85, 7833 (1999).

[3] S. Gama et al., Phys. Rev. Lett. 93, 237202 (2004).

[4] M. Tanaka et al., Appl. Phys. Lett. 65, 1964 (1994).

[5] M. Ramsteiner et al., Phys. Rev. B 66, 081304(R) (2002).

[6] K. Motizuki and K. Katoh, J. Phys. Soc. Jpn. 53, 735 (1984).

[7] V. M. Kaganer et al., Phys. Rev. Lett. 85, 341 (2000).

[8] T. Plake et al., Appl. Phys. Lett. 80, 2523 (2002).

[9] M. Kästner et al., J. Appl. Phys. 92, 5711 (2002).

[10] F. Iikawa et al., Appl. Phys. Lett. 85, 2250 (2004).

[11] N. Menyuk et al., Phys. Rev. 177, 942 (1969).

[12] S. Sanvito and N. A. Hill, Phys. Rev. B 62, 15553 (2000).

[13] F. Schippan et al., J. Vac. Sci. Technol. B 17, 1716 (1999).

[14] M.P. F. Godoy et al., Rev. Sci. Instrum. 75, 1947 (2004).

[15] B. T. M. Willis and H.P. Rooksby, Proc. Phys. Soc. London, Sect. B 67, 290 (1954).

[16] M. Dörfler and K. Bärner, Phys. Status Solidi A 17, 141 (1973).

[17] J.M. Soler et al., J. Phys. Condens. Matter 14, 2745 (2002).

[18] J. P. Perdew, K. Burke, and M. Ernzerhof, Phys. Rev. Lett. 77, 3865 (1996).

[19] I. Rungger and S. Sanvito (to be published).

[20] T. Kato, K. Nagai, and T. Aisaka, J. Phys. C 16, 3183 (1983). 